\title{
Papers
}

\section{Cohort study of sibling effect, infectious diseases, and risk of atopic dermatitis during first 18 months of life}

\author{
Christine Stabell Benn, Mads Melbye, Jan Wohlfahrt, Bengt Björkstén, Peter Aaby
}

\begin{abstract}
Objectives To determine whether early infectious diseases could explain the association between number of siblings and other markers of microbial exposure and the development of atopic dermatitis before the age of 18 months.

Design Cohort study. Information on atopic dermatitis, infectious diseases occurring before 6 months of age, number of siblings, early day care, pet keeping, farm residence, and background factors was collected in telephone interviews. Setting Danish national birth cohort.

Participants 24341 mother-child pairs.

Main outcome measures Incidence rate ratios of atopic dermatitis.
\end{abstract}

Results 13070 children (54\%) had at least one clinically apparent infectious disease before 6 months of age. At age 18 months, $2638(10.8 \%)$ of the children had had atopic dermatitis. The risk of atopic dermatitis increased with each infectious disease before 6 months of age (incidence rate ratio 1.08, 95\% confidence interval 1.04 to 1.13). The risk of atopic dermatitis decreased with each additional exposure to three or more siblings, day care, pet ownership, and farm residence $(0.86,0.81$ to 0.93 ).

Conclusions Early infections do not seem to protect against allergic diseases. The protective effect of number of siblings, day care, pet ownership, and farm residence remained after adjustment for clinically apparent infectious diseases, suggesting that the effect is established independently early in life.

\section{Introduction}

Epidemiological studies have consistently shown an inverse relation between number of siblings and allergic diseases. ${ }^{1}$ Strachan formulated the hygiene hypothesis, suggesting that the risk of allergic disease is reduced by infectious diseases in infancy transmitted by older siblings. ${ }^{2}$ However, though factors associated with microbial exposure, such as early day care, pet keeping, and living on a farm, have likewise been associated with decreased risk of allergic diseases, the association between infectious diseases and allergic diseases has not been consistent. ${ }^{3-12}$

We investigated whether a protective effect of infectious diseases during the first 6 months of life could explain the association between number of siblings and other markers of microbial exposure and the development of atopic dermatitis before 18 months of age.

\begin{abstract}
Methods
The study was based on mother-child pairs enrolled in the national birth cohort in Denmark, which comprised consecutively recruited pregnant women from 1997 to 2002. ${ }^{13}$ All women who spoke Danish and intended to carry their pregnancy to term were invited to participate when they first consulted their general practitioner about the pregnancy. Women were invited to take part in four computer-assisted telephone interviews at 12 and 30 weeks' gestation (interviews 1 and 2) and when the child was 6 and 18 months old (interviews 3 and 4). The women were called up to four times for each interview. If a woman was not at home and available for interview, it was cancelled. Less than $2 \%$ of the women enrolled refused to take part in an interview. In April 2000 detailed questions about itchy rash and atopic dermatitis were integrated into the fourth interview. Inclusion criteria for the present study were participation in this modified interview as well as all the previous interviews. When our study was initiated in November 2002, 44779 women were due to have taken part in the modified fourth interview. However, 20438 had not completed all four interviews. We enrolled the 24341 remaining pairs. Data on day care and exclusive breastfeeding were available for 15430 mother-child pairs who took part in the third interview after April 2000.
\end{abstract}

\section{Atopic dermatitis}

In the fourth interview, the mothers were asked about itchy rash and atopic dermatitis in their child and when it had occurred. As described elsewhere, a dermatologist examined a subgroup of the children, and the combination of answers that resulted in the highest sum of sensitivity and specificity was determined..$^{14}$ Cases of atopic dermatitis were defined as children whose mothers reported that the child had had an itchy rash or atopic dermatitis diagnosed by a doctor, or both, and recurrent rash or rash in at least four consecutive half month periods, or both, and relevant localisation of the rash in elbow and knee creases, on the hands, on the face, or in at least four places or generalised. ${ }^{14}$

\section{Data on infectious diseases and use of antibiotics}

In the fourth interview, the mothers were asked whether the child had had colds and diarrhoea, if these had lasted more than three days, and when they had occurred. They were also asked if the child had had otitis media, pneumonia, or other diseases (if so, which) and when they had occurred. We obtained date and diagnoses for admissions to hospital for infectious diseases from the national hospital discharge register. A variable for any type of infectious disease before 6 months of age was created. Furthermore, the mothers were asked about use of antibiotics in the child during the first 6 months of life. 


\section{Markers of microbial exposure}

We obtained from the interviews data on number of siblings, pet keeping, farm residence, and, for a subgroup, day care before 6 months of age. Their effect was investigated separately and combined, defined as microbial exposure. The effect of number of siblings was explored as a classified variable with $0,1,2,3$, or $\geq 4$ siblings. When we included this variable in the models as a confounder, and in the analyses combining the four variables, we grouped it into having $\geq 3$ siblings or fewer.

\section{Data on background factors}

From the interviews we determined the date of birth and sex of the child and, for a subgroup, information on breast feeding. For parents, we determined the date of birth of the mother and details of allergic diseases (asthma, hay fever, atopic dermatitis), occupational class, educational level, smoking, cohabitation, and household income. Data on birth characteristics were obtained from the Danish medical birth registry.

\section{Statistical methods}

We used $\chi^{2}$ tests to analyse the prevalence ratios for having had an infectious disease at 6 months of age according to characteristics of the child and its family. We used Cox proportional hazards models with age as the underlying time (that is, estimated non-parametric) and reported incidence rate ratios to examine the effect of infectious diseases on atopic dermatitis. We retained in the model any variables (of sex, parental history of allergy, number of siblings, maternal education, and background factors) that changed the estimate of the incidence rate ratio for atopic dermatitis by more than $5 \%$. Potential effect modification was investigated by testing the homogeneity of the effect of infectious diseases within different strata. When we analysed the data according to sex, we became aware of a significant interaction between sex, age, and atopic dermatitis: more boys than girls had atopic dermatitis in the first months of life, whereas this was not the case later. Subsequently we controlled for this in all analyses where results were different by time and sex. Furthermore, we presented the main associations separately for boys and girls.

\section{Results}

Overall $13070(54 \%)$ of 24341 children had at least one clinically apparent infectious disease before 6 months of age, most of them $(85 \%)$ having a cold. Boys had slightly more infectious diseases than girls $(55 \% v 52 \%, \mathrm{P}<0.001)$. Table 1 shows factors associated with having an infectious disease during the first 6 months of life.

A total of 2638 children had had atopic dermatitis by the age of 18 months; $1474(56 \%)$ boys and $1164(44 \%)$ girls. Overall, having had at least one infectious disease before 6 months of age was associated with atopic dermatitis (incidence rate ratio 1.09, $95 \%$ confidence interval 1.00 to 1.19 ). Table 2 gives details of diseases reported for more than 250 children before 6 months of life. All tended to be associated with atopic dermatitis. The association increased in strength with number of infectious diseases (table 2). We found no evidence of interaction between infectious disease and family history of allergic diseases (data not shown). Adjustment for exclusive breastfeeding for four months did not change the results (data not shown). Use of antibiotics during the first 6 months of life was not associated with atopic dermatitis (1.09, 0.93 to 1.27), even when we controlled for infectious diseases (1.07, 0.91 to 1.25), and use of antibiotics did not change the estimates of the effect of infectious diseases (data not shown).

We explored the effects of markers of microbial exposure, previously associated with protection against allergic diseases, in
Table 1 Prevalence ratios of having at least one infectious disease during first six months of life according to characteristics of child and its family

No with infection (\%) Prevalence ratio for infection $(95 \%$

\begin{tabular}{|c|c|c|}
\hline \multicolumn{3}{|l|}{ Sex of child: } \\
\hline Girl & $6187(52)$ & 1 (ref) \\
\hline Boy & $6883(55)$ & $1.07 \quad(1.05$ to 1.10$)$ \\
\hline \multicolumn{3}{|c|}{ Parents with allergic disease: } \\
\hline 0 & $6015(53)$ & 1 (ref) \\
\hline 1 & $5537(54)$ & 1.02 (1.00 to 1.05$)$ \\
\hline 2 & $1518(58)$ & $1.10 \quad(1.06$ to 1.14$)$ \\
\hline \multicolumn{3}{|c|}{ Maternal education (years): } \\
\hline $6-10$ & $4482(54)$ & 1 (ref) \\
\hline$\geq 11$ & $8586(53)$ & $0.98 \quad(0.96$ to 1.00$)$ \\
\hline \multicolumn{3}{|c|}{ Maternal age (years): } \\
\hline$<25$ & $1064(53)$ & $0.99 \quad$ (0.95 to 1.04$)$ \\
\hline $25-29$ & $5001(54)$ & $1 \quad$ (ref) \\
\hline $30-34$ & $5181(56)$ & 1.04 (1.01 to 1.06$)$ \\
\hline$>35$ & $1824(49)$ & $0.90 \quad(0.87$ to 0.94$)$ \\
\hline \multicolumn{3}{|c|}{ Season of birth: } \\
\hline Spring & $3160(49)$ & $1.03 \quad$ (0.99 to 1.07$)$ \\
\hline Summer & $3969(59)$ & $1.24 \quad$ (1.20 to 1.28$)$ \\
\hline Autumn & $3082(61)$ & $1.30 \quad$ (1.26 to 1.35$)$ \\
\hline Winter & $2859(47)$ & $1 \quad$ (ref) \\
\hline \multicolumn{3}{|c|}{ No of older siblings: } \\
\hline 0 & $4889(47)$ & $1 \quad$ (ref) \\
\hline 1 & $5836(61)$ & $1.30 \quad$ (1.27 to 1.33$)$ \\
\hline 2 & $1942(55)$ & $1.17 \quad$ (1.13 to 1.21$)$ \\
\hline 3 & $348(53)$ & 1.12 (1.04 to 1.21$)$ \\
\hline$\geq 4$ & $55(42)$ & $0.89 \quad(0.73$ to 1.10$)$ \\
\hline \multicolumn{3}{|c|}{ Farm residence: } \\
\hline Yes & $970(53)$ & $0.99 \quad$ (0.94 to 1.03$)$ \\
\hline No & $12100(54)$ & $1 \quad$ (ref) \\
\hline \multicolumn{3}{|l|}{ Pet keeping: } \\
\hline Yes & $4681(45)$ & $0.98 \quad(0.95$ to 1.01$)$ \\
\hline No & $6463(46)$ & $1 \quad$ (ref) \\
\hline \multicolumn{3}{|c|}{ Day care at 6 months*: } \\
\hline Yes & $1095(57)$ & $1.10 \quad$ (1.06 to 1.15$)$ \\
\hline No & $7041(52)$ & $1 \quad$ (ref) \\
\hline
\end{tabular}

* Subgroup analysis based on 15430 children for whom information on day care was available.

a multivariable model with and without adjustment for infectious diseases (table 3). Farm residence, number of siblings, pet keeping, and early day care all tended to be inversely associated with risk of atopic dermatitis, with and without adjustment for infectious diseases. The overall incidence rate ratio of atopic dermatitis associated with having three or more siblings was 0.79 (0.62 to 1.01). The estimates of atopic dermatitis occurring between 0 and 6 months of age $(0.76,0.53$ to 1.09$)$ and between 6 and 18 months of age $(0.82,0.58$ to 1.15$)$ were similar. We had data on early day care for 15430 children. In a separate analysis, we created a score of factors indicative of microbial exposures (having three or more siblings, farm residence, pet keeping, and early day care). After adjustment for number of infectious diseases, the number of factors was inversely associated with atopic dermatitis, the effect per factor being 0.86 (0.81 to 0.93 ) (table 3). In the same model, the additional effect for each infectious disease was 1.09 (1.03 to 1.14).

The effect of infectious diseases was stronger in girls than in boys (table 2). There was no sex difference in the effect of number of siblings, pet keeping, farm residence, or early day care (data not shown). 
Table 2 Adjusted incidence rate ratios (IRR) of atopic dermatitis (AD) according to type of early infection

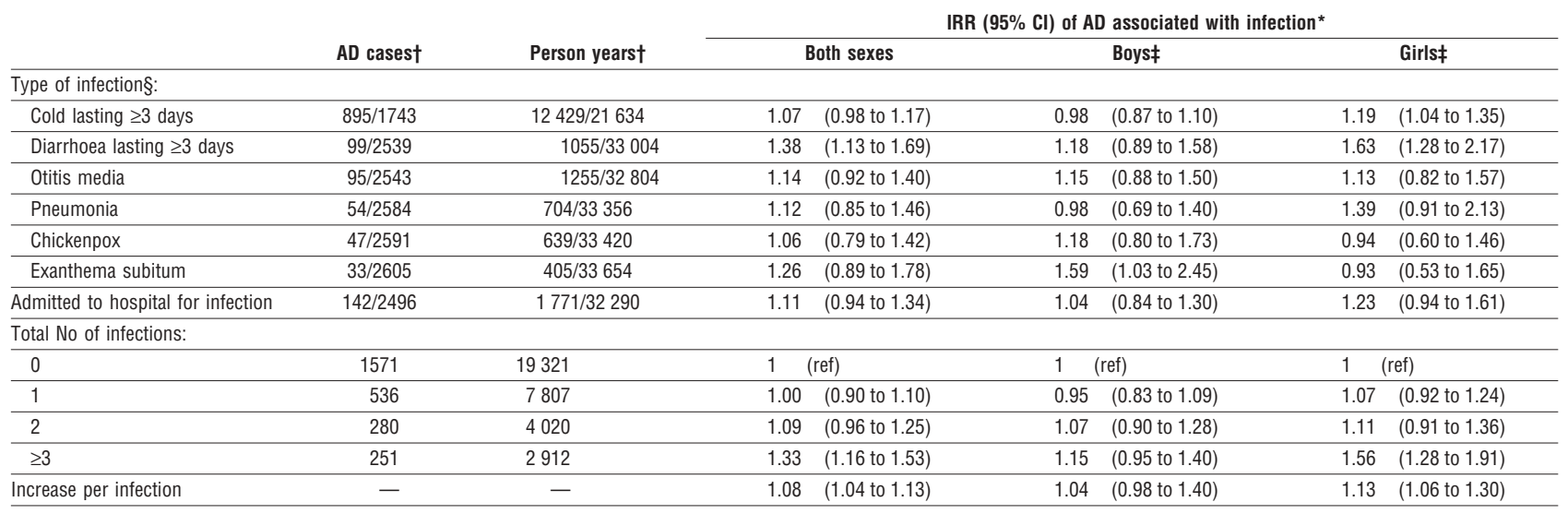

${ }^{*}$ Adjusted for parental history of allergy, sex, number of siblings, season of birth, maternal age, and maternal education.

†Figures are exposed/not exposed, unless indicated otherwise.

fObtained in independent analyses. Adjusted for parental history of allergy, number of siblings, season of birth, maternal age, and maternal education.

$\S$ Data on specific infections presented for diseases reported by more than 250 children.

\section{Discussion}

\section{Main findings}

Infectious diseases in the first 6 months of life are associated with an increased risk of atopic dermatitis, while the opposite is true for several environmental factors indicative of microbial exposure. Our findings support the importance of microbial exposure for preventing atopic dermatitis but challenge the hypothesis that infectious diseases in infancy protect against its development.

\section{Strengths and limitations of the study}

The strengths of our study include the large sample size and the population based cohort design. Information on all types of infectious disease and symptoms of atopic dermatitis was obtained in early infancy. The diagnosis of atopic dermatitis was based on a set of diagnostic criteria, which we have found to be robust and reasonably specific. ${ }^{14}$ The observed cumulative prevalence of $10.8 \%$ is lower than reported in other cohort studies of children of similar ages. However, many cohorts tend to contain more children with risk factors for allergy or with atopic derma- titis defined by the mother or diagnosed by a doctor; both diagnoses being non-specific. ${ }^{15}$ Because of the large sample size we were able to control for numerous potential confounders. Importantly, we conducted the analyses as survival analyses including only infectious diseases occurring before the appearance of atopic dermatitis. We addressed the effect of all reported early infectious diseases on the risk of an allergic disease in a population with an evident sibling effect, while previous similar studies have addressed only the potential effect of subgroups of infectious diseases. In such studies, the presence of systemic childhood diseases could not explain the protective effect of siblings on allergic diseases ${ }^{4}$ and asthma, ${ }^{6}$ though a protective effect of measles infection was observed. Seropositivity to hepatitis A virus, Helicobacter pylori, and Toxoplasma gondii seemed to explain the sibling effect to some extent in one study ${ }^{10}$ but not in another. ${ }^{5}$ Neonatal infectious disease could not explain an observed sibling effect on hay fever. ${ }^{7}$ Furthermore, respiratory tract infections could not explain the sibling effect on atopy, rhinitis, ${ }^{11}$ or asthma. ${ }^{9}$ Finally, infectious diseases in infancy that resulted in the child being seen by a general practitioner could

Table 3 Adjusted incidence rate ratios (IRR) of atopic dermatitis (AD) associated with factors indicative of microbial exposure.

\begin{tabular}{|c|c|c|c|c|}
\hline \multirow[b]{2}{*}{ Exposure } & \multirow[b]{2}{*}{$A D$ cases $†$} & \multirow[b]{2}{*}{ Person years $\dagger$} & \multicolumn{2}{|c|}{$\operatorname{IRR}(95 \% \mathrm{Cl})$ of $\mathrm{AD}^{*}$} \\
\hline & & & Unadjusted & Adjusted for infections \\
\hline \multicolumn{5}{|l|}{ No of siblings: } \\
\hline 0 & 1112 & 14621 & (ref) & 1 (ref) \\
\hline 1 & 1096 & 13339 & $1.06 \quad$ (0.97 to 1.15) & $1.03 \quad$ (0.94 to 1.13$)$ \\
\hline 2 & 364 & 4973 & $0.96 \quad$ (0.84 to 1.09) & $0.94 \quad(0.82$ to 1.07$)$ \\
\hline 3 & 59 & 939 & $0.88 \quad$ (0.67 to 1.15$)$ & $0.86 \quad$ (0.66 to 1.13$)$ \\
\hline 4 & 7 & 188 & $0.53 \quad$ (0.25 to 1.12) & $0.53 \quad(0.25$ to 1.11$)$ \\
\hline Decrease per sibling & - & - & $0.97 \quad$ (0.92 to 1.03$)$ & $0.96 \quad$ (0.91 to 1.02$)$ \\
\hline Farm residence & $170 / 2668$ & $2584 / 31476$ & $0.95 \quad$ (0.81 to 1.11$)$ & $0.90 \quad(0.74$ to 1.10$)$ \\
\hline Pet keeping & $987 / 1651$ & $14600 / 19460$ & $0.87 \quad(0.80$ to 0.94$)$ & $0.87 \quad$ (0.79 to 0.96$)$ \\
\hline Day care before 6 months $\dagger$ & $181 / 1589$ & $2690 / 18806$ & $0.82 \quad$ (0.70 to 0.96$)$ & $0.82 \quad$ (0.70 to 0.96$)$ \\
\hline \multicolumn{5}{|l|}{ Total No of exposuresł: } \\
\hline 0 & 957 & 10134 & $1 \quad$ (ref) & $1 \quad$ (ref) \\
\hline 1 & 633 & 8603 & $0.83 \quad(0.75$ to 0.92$)$ & $0.83 \quad(0.75$ to 0.92$)$ \\
\hline 2 & 161 & 2459 & $0.77 \quad(0.65$ to 0.91$)$ & (0.65 to 0.91$)$ \\
\hline$\geq 3$ & 19 & 297 & $0.74 \quad$ (0.47 to 1.17$)$ & $0.74 \quad$ (0.47 to 1.17$)$ \\
\hline Decrease per exposure & - & - & $0.86 \quad$ (0.81 to 0.93$)$ & (0.81 to 0.93$) \S$ \\
\hline
\end{tabular}

*Obtained in multivariable model including all variables at same time. Adjusted for parental history of allergy, sex, season of birth, maternal age, and maternal education.

tFigures are exposed/not exposed, unless indicated otherwise.

‡Subgroup analysis based on 15430 children for whom information on day care was available.

$\S$ Boys: 0.85 (0.78 to 0.94 ), girls: 0.88 ( 0.79 to 0.97 ) 
not explain a sibling effect on allergic diseases diagnosed by a doctor. $^{12}$

\section{Possible explanations of the findings}

Most of the infectious diseases occurring in our population were respiratory tract infections and diarrhoea. Systemic childhood diseases, such as measles, may be more likely to have long lasting effects on the immune system. However, we found evidence for a sibling effect before 6 months of age. Hence, systemic childhood diseases, which mostly occur later in childhood, cannot explain the sibling effect.

The tendency for reduced risk associated with number of siblings, early day care, pet keeping, and farm residence could reflect microbial stimulation by environmental micro-organisms or microbial products, or both, which does not result in clinically apparent disease, but anyhow stimulates the immune system. Such stimulation may take place in the gut, where the permanent and enormous load ensures a constant source of stimulation. ${ }^{16-19}$

The inverse association between number of siblings and risk of atopic dermatitis early in life and the lack of effect of infectious diseases on allergic diseases could suggest a "parity effect" originating in utero. Several studies have found evidence for altered immune function as a function of birth order. ${ }^{20}{ }^{21}$ However, even if the sibling effect originated in utero, it would explain neither the increasing incidence of allergic diseases in recent years nor the effect of the indicators of microbial exposure.

We measured clinically apparent infections and not merely exposure to microbes. Previously, it was noted that adults with atopic dermatitis had a higher incidence of recurrent cold sores and upper respiratory tract infections, which led to the suggestion that this could be due to dysfunctioning cell mediated immunity. ${ }^{22}$ A recent study reported concomitant diseases in children with atopic dermatitis. ${ }^{23}$ The observed association between clinically apparent infectious diseases and atopic dermatitis may be caused by an underlying immature immune system.

Overall, boys had more infectious diseases and more atopic dermatitis, but these were associated only in girls. Interestingly, a Swedish group recently reported pneumonia to be significantly more common in girls with atopic dermatitis than in girls without, whereas this was not the case in boys. ${ }^{23}$

\section{Conclusions}

In conclusion, we found that infectious diseases occurring early in life were associated with an increased risk of atopic dermatitis before 18 months. This contrasts with the previously held belief of a protective effect of early infections on the development of allergic diseases. The inverse association between atopic dermatitis and number of siblings, early day care, pet keeping, and farm residence remained after we controlled for number of clinically apparent infectious diseases, suggesting that these effects are mediated early in life and independently of clinically apparent infectious diseases.

Contributors: $\mathrm{CSB}, \mathrm{MM}, \mathrm{BB}$, and PA were involved in drafting the study protocol. CSB and JW analysed the data. All authors were involved in interpreting results and writing the paper. CSB, MM, and PA are the guarantors. Funding: Danish National Research Foundation, Pharmacy Foundation of 1991, Egmont Foundation, March of Dimes Birth Defects Foundation, Augustinus Foundation, Leo Foundation, and Aage Bang's Foundation.

Competing interests: None declared.

Ethical approval: Ethical committees in Denmark and by the Data Protection Board. The steering committee for the Danish National Research Foundation approved the use of data for the present study.

1 Karmaus W, Botezan C. Does a higher number of siblings protect against the development of allergy and asthma? A review. J Epidemiol Community Health 2002;56:209-17.
What is already known on this topic

The risk of atopic diseases decreases with exposure to siblings, early day care, living on a farm, and pet keeping

Infectious diseases early in life may protect against the development of atopic diseases

What this study adds

Clinically apparent infectious diseases do not protect against the development of atopic diseases

The protective effect of siblings, as well as that of early day care, living on a farm, and pet keeping, is mediated independently of clinically apparent infections in the first 6 months of life

2 Strachan DP. Hay fever, hygiene and household size. BMJ 1989;299:1259-60.

Strachan DP. Family size, infection and atopy: the first decade of the "hygiene hypothesis". Thorax 2000;55(suppl 1):S2-10.

Bodner C, Godden D, Seaton A. Family size, childhood infections and atopic diseases. The Aberdeen WHEASE Group. Thorax 1998;53:28-32.

5 Linneberg A, Østergaard C, Tvede M, Andersen LP, Nielsen NH, Madsen F, et al. IgG antibodies against microorganisms and atopic disease in Danish adults: the Copenhagen allergy study. J Allergy Clin Immunol 2003;111:847-53.

6 Wickens KL, Crane J, Kemp TJ, Lewis SJ, D'Souza WJ, Sawyer GM, et al. Family size, infections, and asthma prevalence in New Zealand children. Epidemiology 1999;10:699infection.

7 Strachan DP, Taylor EM, Carpenter RG. Family structure, neonatal infection, and hay fever in adolescence. Arch Dis Child 1996;74:422-6.

8 Forastiere F, Agabiti N, Corbo GM, Dell'Orco V, Porta D, Pistelli R, et al. Socioeconomic status, number of siblings, and respiratory infections in early life as determinants of atopy in children. Epidemiology 1997;8:566-70.

9 Nafstad P, Magnus P, Jaakkola JJ. Early respiratory infections and childhood asthma. Pediatrics 2000;106:E38.

10 Matricardi PM, Rosmini F, Ferrigno L, Nisini R, Rapicetta M, Chionne P, et al. Cross sectional retrospective study of prevalence of atopy among Italian military students with antibodies against hepatitis A virus. BMJ 1997;314:999-1003.

11 Ponsonby AL, Couper D, Dwyer T, Carmichael A, Kemp A. Relationship between early life respiratory illness, family size over time, and the development of asthma and hay fever: a seven year follow up study. Thorax 1999;54:664-9.

12 McKeever TM, Lewis SA, Smith C, Collins J, Heatlie H, Frischer M, et al. Early exposure to infections and antibiotics and the incidence of allergic disease: a birth cohort study with the West Midlands general practice research database. J Allergy Clin Immunol 2002;109:43-50

13 Olsen J, Melbye M, Olsen SF, Sørensen TI, Aaby P, Andersen AM, et al. The Danish national birth cohort-its background, structure and aim. Scand J Public Health 2001;29:300-7.

14 Benn CS, Benfeldt E, Andersen PK, Olesen AB, Melbye M, Björkstén B. Atopic dermatitis in young children: diagnostic criteria for use in epidemiological studies based on telephone interviews. Acta Derm Venereol 2003;83:347-50.

15 Harris JM, Cullinan P, Williams HC, Mills P, Moffat S, White C, Taylor AJN. Environmental associations with eczema in early life. Br J Dermatol 2001;144:795-802.

16 Matricardi PM, Ronchetti R. Are infections protecting from atopy? Curr Opin Allergy Clin Immunol 2001;1:413-9.

17 Strannegård Ö, Strannegård IL. The causes of the increasing prevalence of allergy: is atopy a microbial deprivation disorder? Allergy 2001;56:91-102.

18 Kalliomäki M, Isolauri E. Role of intestinal flora in the development of allergy. Curr Opin Allergy Clin Immunol 2003;3:15-20.

19 Björkstén B. The intrauterine and postnatal environments. J Allergy Clin Immunol 1999;104:1119-27.

20 Karmaus W, Arshad H, Mattes J. Does the sibling effect have its origin in utero? Investigating birth order, cord blood immunoglobulin E concentration, and allergic sensitization at age 4 years. Am J Epidemiol 2001;154:909-15.

21 Devereux G, Barker RN, Seaton A. Antenatal determinants of neonatal immune responses to allergens. Clin Exp Allergy 2002;32:43-50.

22 Rystedt I, Strannegård IL, Strannegård Ö. Recurrent viral infections in patients with past or present atopic dermatitis. Br J Dermatol 1986;114:575-82.

23 Böhme M, Lannerö E, Wickman M, Nordvall SL, Wahlgren CF. Atopic dermatitis and concomitant disease patterns in children up to two years of age. Acta Derm Venereol 2002;82:98-103

(Accepted 5 March 2004)

doi $10.1136 /$ bmj.38069.512245.FE

Department of Epidemiology Research, Danish Epidemiology Science Centre,

Department of Epidemiology Re

Statens Serum Institut, Denmark
Christine Stabell Benn research fellow

Mads Melbye professor of epidemiology

Jan Wohlfahrt statistician

Projecto de Saúde de Bandim, Bissau, Guinea-Bissau

Peter Aaby professor of international health 
Center for Allergy Research and Institute of Environmental Medicine, Karolinska Institutet, Stockholm, Sweden

Correspondence to: C Benn cb@ssi.dk

Bengt Björkstén professor of paediatrics and allergy prevention 